\title{
1 Multiple drug resistance in hookworms infecting greyhound dogs in the
}

\section{USA}

3 Pablo D. Jimenez Castro ${ }^{1,2 *}$, Abhinaya Venkatesan ${ }^{3}$, Elizabeth Redman ${ }^{3}$, Rebecca Chen ${ }^{3}$,

4 Abigail Malatesta ${ }^{1}$, Hannah Huff ${ }^{1}$, Daniel A. Zuluaga Salazar ${ }^{4}$, Russell Avramenko ${ }^{3}$, John. S.

5 Gilleard $^{3}$, Ray M. Kaplan ${ }^{1}$

6

$7 \quad{ }^{1}$ Department of Infectious Diseases, College of Veterinary Medicine, University of

8 Georgia, Athens, GA 30602, USA

$9 \quad{ }^{2}$ Grupo de Parasitología Veterinaria, Universidad Nacional de Colombia

$10{ }^{3}$ Department of Comparative Biology and Experimental Medicine, Faculty of Veterinary

11 Medicine, Host-Parasite Interactions Program, University of Calgary, Alberta, Canada

12 4aboratorio de Parasitología Veterinaria, Corporación Universitaria Santa Rosa de Cabal-

13 UNISARC, Santa Rosa de Cabal, Risaralda, Colombia

$14 *$ Corresponding Author. Tel: 706-542-0742

15 Email: pdj38559@uga.edu

16 Address: Wildlife Health Building, College of Veterinary Medicine, University of Georgia,

17 Athens, GA 30602, USA

18

19 


\section{Abstract}

24 The hookworm Ancylostoma caninum is the most prevalent nematode parasite of dogs.

25 Recently, we confirmed multiple-drug resistance (MDR) in several A. caninum isolates to

26 all anthelmintic drug classes approved for the treatment of hookworms in dogs in the

27 United States (USA). Cases of MDR hookworms appear to be highly overrepresented in

28 greyhounds, suggesting that the MDR worms evolved on racing greyhound farms/kennels.

29 The aims of this study were to evaluate the range of drug-resistant phenotypes and

30 genotypes of the A. caninum infecting greyhounds. Fecal samples from recently retired

31 greyhounds originating from geographically diverse areas of the USA were acquired from

32 two greyhound adoption kennels, one active greyhound racing kennel, and three veterinary

33 practices that work with adoption kennels. Fecal egg counts (FECs) were performed on

34 fecal samples from 219 greyhounds, and despite almost all the dogs having been treated

35 with one or more anthelmintics in the previous two to four weeks, the mean FEC was 822.4

36 eggs per gram (EPG). Resistance to benzimidazoles and macrocyclic lactones were

37 measured using the egg hatch assay (EHA) and the larval development assay (LDA)

38 respectively. We performed 23 EHA and 22 LDA on either individual or pooled feces,

39 representing 81 animals. Mean and median $\mathrm{IC}_{50}$ and $\mathrm{IC}_{95}$ values for the EHA were $5.3 \mathrm{uM}$,

$403.6 \mathrm{uM}$, and $24.5 \mathrm{uM}, 23.4 \mathrm{uM}$ respectively. For the LDA, mean and median $\mathrm{IC}_{50}$ values

41 were $749.8 \mathrm{nM},>1000 \mathrm{nM}$ respectively. These values range from 62 to 68 times higher

42 than those we measured in our susceptible laboratory isolates. Pre-treatment fecal samples

43 could not be obtained, however, post-treatment samples representing 219 greyhounds were

44 collected. For samples collected <10 days post-treatment with albendazole, moxidectin, or

45 a combination of febantel-pyrantel-moxidectin, the mean FEC were 349, 333, and 835

46 EPG, respectively. Samples collected 10-21 days post-treatment with albendazole, 
47 moxidectin, or pyrantel, yielded mean FEC of 1874, 335, and 600 EPG, respectively.

48 Samples collected $>21$ days post-treatment with albendazole or moxidectin yielded mean

49 FEC of 1819 and 1117 EPG, respectively. We obtained DNA from hookworm eggs isolated

50 from 70 fecal samples, comprised of 60 individual dogs and 10 pools from multiple dogs.

51 Deep sequencing of the isotype $1 \beta$-tubulin gene revealed the presence of the F167Y

52 (TTC>TAC) resistance polymorphism in $99 \%$ of these samples, with $69 \%$ having $\geq 75 \%$

53 resistant allele frequency. No resistance-associated polymorphisms were seen at any of the

54 other $\beta$-tubulin codons previously reported as associated with benzimidazole resistance in

55 Strongylid nematodes. These clinical, in vitro, and genetic data provide strong evidence

56 that racing and recently retired greyhound dogs in the USA are infected with MDR $A$.

57 caninum at very high levels in terms of both prevalence and infection intensity.

Keywords: Ancylostoma caninum, hookworms, multiple-drug resistance (MDR), deep-

60 amplicon, greyhounds

61

\section{Introduction}

The canine hookworm, Ancylostoma caninum is the most prevalent and important

64 intestinal nematode parasite of dogs in the USA, with the prevalence depending on age,

65 level of care and geographic location of the dog (Little et al., 2009). A recent study

66 evaluating over 39 million fecal samples from 2012-2018, found that the prevalence of

67 hookworms remained very stable from 2012-2014 at around 2\%, but then from 2015

68 onwards, there was a steady yearly increase, with an overall increase of $47 \%$ by 2018

69 (Drake and Carey, 2019). Moreover, in a study assessing intestinal parasites from 3006 dog 
70 fecal samples collected in 288 off-leash dog parks across the USA in 2019, the prevalence

71 of A. caninum was $7.1 \%$ (Stafford et al., 2020). Interestingly, this prevalence is more than

72 twice as high as that reported for 2018 by (Drake and Carey, 2019), and is more than 70\%

73 higher than the mean prevalence for 2017-2019 reported by (Sweet et al., 2021). Taken

74 together these data suggest that hookworm prevalence is rapidly increasing, and that dogs

75 that visit dog parks are at a higher risk of infection.

76 Anthelmintic drugs currently approved for the treatment of A. caninum in the United

77 States include, febantel and fenbendazole, moxidectin and milbemycin oxime, and pyrantel,

78 of the benzimidazole, avermectin/milbemycin, and tetrahydropyrimidine classes,

79 respectively. In registration studies, febantel, moxidectin and milbemycin oxime all

80 demonstrated efficacies of $>99 \%$ (F.D.A, 1994, 1998, 2006), fenbendazole demonstrated

81 efficacy of $>98 \%$ (F.D.A, 1983) and pyrantel demonstrated a slightly variable efficacy,

82 with a mean across studies of approximately 94\%, where more than half of those studies

83 yielded $>99 \%$ (F.D.A, 1993).

84 Hookworms are blood-feeding nematodes that use a cutting apparatus to attach to

85 the intestinal mucosa and submucosa, and contract their muscular esophagus to create

86 negative pressure, which sucks a plug of tissue into their buccal capsules (Hotez et al.,

87 2004). Bleeding is facilitated by both mechanical damage and chemical action by

88 hydrolytic enzymes that cause rupture of capillaries and arterioles (Stassens et al., 1996).

89 Pathological consequences of infection in dogs include iron-deficiency anaemia,

90 hypoalbuminemia, and an enteritis characterized by diarrhoea, that may contain fresh

91 (haematochezia) or digested blood (melena) (Epe, 2009; Kalkofen, 1987; Taylor et al., 92 2016). 
In the past few years, there is empirical evidence that veterinarians are diagnosing

94 increasing numbers of cases of persistent hookworm infections, primarily in greyhound

95 dogs, that appear refractory to typical anthelmintic therapy. Recent work in our laboratory

96 confirmed that many, if not most, of these persistent hookworm cases are likely due to

97 multiple-drug resistance (MDR) in A. caninum, with retired racing greyhounds highly over-

98 represented among the cases reported to our laboratory. Our laboratory established one of

99 these A. caninum isolates (Worthy), which we obtained from a recently adopted retired

100 racing greyhound dog. In a controlled efficacy study, we confirmed high levels of

101 resistance to all classes of drugs approved for treatment of hookworm in dogs;

102 fenbendazole, pyrantel pamoate and milbemycin oxime yielded efficacies of $26 \%, 23 \%$ and

103 9\%, respectively (Jimenez Castro et al., 2020). A. caninum is also zoonotic in humans, and

104 MDR A. caninum will not respond to usual anthelmintic treatments administered by

105 physicians.

106 Greyhound racing was once very trendy and profitable in the USA. At the sport's

107 peak in 1991, dog racing was rated the sixth most popular sporting activity in the USA, was

108 legal in 19 states, and generated around 100,000 jobs with wager revenues of $\$ 3.5$ billion

109 USD (Theil, 2021). At that time there were 38,000 individual pups and 5,700 registered

110 racers, but by 2020 those numbers had dramatically decreased to 4,300 and 850 ,

111 respectively (Gartland, 2021). Kansas, the state with the most greyhound breeding farms,

112 had 274 greyhound breeding farms in the 1990's, but by 2015 this number had decreased

113 by more than half to 130 (Hall, 2016), and continues to fall. These farms tend to have large

114 dog populations; more than $60 \%$ and $80 \%$ of all racing greyhounds come from farms with

$115>250$ dogs and >100 dogs, respectively (Hall, 2016). 
117 amendment which banned wagering on live dog races, including greyhound racing in the

118 state as of January 1st, 2021 (State, 2018). In 2018, 65\% of the greyhound tracks in the

119 USA were in Florida. However, with the closure of these tracks and others in several other 120 states in the past few years, to our knowledge there currently are only 7 tracks in 5 states

121 remaining. This change likely represents the beginning of the end for greyhound racing in

122 the USA. In parallel, a large network of greyhound adoption groups has been active for

123 many years, having over 160 organizations across the USA and Canada with the majority

124 being in FL followed by $\mathrm{NY}$ and $\mathrm{OH}$. This results in thousands of retired racing 125 greyhounds being adopted as pets every year (Lord et al., 2007), and with the demise of the 126 greyhound racing industry, the number of adoptions are rapidly increasing. Thus, it is

127 important for the health of both racing greyhounds and pet dogs to determine the extent of 128 the MDR hookworm problem in racing greyhounds.

129 The aims of this study were to investigate the prevalence of infection, the range of 130 in vitro and in vivo drug susceptible/resistant phenotypes, and the frequency of 131 benzimidazole-resistant beta-tubulin genotypes in greyhound dogs infected with $A$. 132 caninum.

\section{2. Materials and Methods}

\subsection{Sample collection}

From February 2019 to February 2020, fecal samples were acquired from two 136 greyhound adoption kennels located in Birmingham, AL, and Dallas, TX, one active

137 greyhound racing kennel in Sanford, FL, and three veterinary practices located in Acworth, 138 GA, Columbia, SC, and St. Petersburg, FL USA that work with greyhound adoption 
139 organizations. Most samples were collected from individual dogs, but from the Sanford, FL

140 site, only anonymous samples from the ground were available. The dogs residing in these

141 kennels originated from 16 different locations in 8 different states. These included five

142 breeding farms located in $\mathrm{KS}, \mathrm{CO}, \mathrm{AR}, \mathrm{TX}$, or $\mathrm{OK}$, and 11 racing tracks located in $\mathrm{AL}$, FL, 143 AR, or WV.

\subsection{In vitro assays}

145 To evaluate drug response phenotypes, the egg hatch assay (EHA) and larval development

146 assay (LDA) were used for benzimidazoles (BZs), and macrocyclic lactones (MLs), 147 respectively as previously described (Jimenez Castro et al., 2019). The concentration ranges

148 for ivermectin aglycone $(1.9-1000 \mathrm{nM})$ in the LDA, and thiabendazole $(0.075-40 \mu \mathrm{M})$ in

149 the EHA were selected based on our previous work to permit the discrimination of

150 susceptible vs resistant isolates of A. caninum (Jimenez Castro et al., 2019). Eggs were

151 isolated using $50 \mathrm{ml}$ tubes containing charcoal granules $(0.5-1 \mathrm{~cm})$ and specialized lids

152 containing a filter that could attach to $15 \mathrm{ml}$ centrifuge tubes. Five to ten grams of feces and

153 approximately $15 \mathrm{ml}$ of water were added to the $50 \mathrm{ml}$ tube containing approximately 5

154 grams of charcoal and vigorously shaken to break up the feces. The lid of the $50 \mathrm{ml}$ tube

155 was removed and replaced with the specialized lid, and a $15 \mathrm{ml}$ tube was attached to the

156 other end. The apparatus was then shaken again which allowed the filtered fecal suspension

157 to fill the $15 \mathrm{ml}$ tube, which was then centrifuged at $240 \mathrm{x} g$ for $10 \mathrm{mins}$. The supernatant

158 was discarded, $10 \mathrm{ml}$ of sodium nitrate (Feca-Med®, Vedco, Inc. St. Joseph; MO, USA

159 specific gravity $=1.2$ ) were added and the tube was vortexed to disperse the fecal material.

160 The tube was then centrifuged again at $240 \times g$ for 10 mins. Following centrifugation, the

161 supernatant containing the eggs was passed through a $20 \mu \mathrm{m}$ sieve, rinsed with distilled 
162 water, transferred to a new tube, and then the volume was adjusted to yield 50-60 eggs per

$16320 \mu \mathrm{l}$ using distilled water. If insufficient eggs were recovered to perform both the EHA 164 and the LDA, then only the EHA was performed.

\subsection{In vivo measurements}

Every dog sampled in this study was treated with anthelmintics at regular intervals;

168 therefore, all of the samples were collected relatively recently post-treatment. Samples were

169 refrigerated immediately after collection and shipped to the Kaplan lab at the University of

170 Georgia in a container with ice packs. In order to account for the differences in timeframe

171 since the previous anthelmintic treatment, dogs were assigned to one of three categories

172 based on the following biological factors: $(\mathrm{A})=<10$ days, as this can be too soon to

173 measure an accurate fecal egg count reduction (FECR) and can lead to false positives due

174 to temporary inhibition of egg production (Jimenez Castro et al., 2019; Jimenez Castro et

175 al., 2020), (B) = $10-21$ days, as this would be an optimal timeframe for measuring the

176 FECR, and $(\mathrm{C})=>21$ days, as there is the possibility that eggs could be shed from

177 reactivated encysted/arrested larvae that migrated to the small intestine and completed 178 development to sexually mature adults following the anthelmintic treatment (due to "larval

179 leak") (Jimenez Castro and Kaplan, 2020). Fecal egg counts (FEC) were performed using 180 the Mini-FLOTAC (University of Naples Federico II, Naples, Italy) procedure with a

181 detection threshold of 5 EPG (Lima et al., 2015; Maurelli et al., 2014), adding two grams of

182 feces to $18 \mathrm{ml}$ of sodium nitrate (Feca-Med®, Vedco, Inc. St. Joseph; MO, USA specific 183 gravity $=1.2$ ). Positive samples were defined as having a FEC of $\geq 5$ EPG. All anthelmintic 184 treatments were administered by either kennel or veterinary practice personnel. Where 
185 products approved for use in dogs were used, treatments were administered according to

186 label instructions; these included febantel-pyrantel pamoate, Drontal ${ }^{\circledR}$ Plus (Elanco,

187 Greenfield, IN), moxidectin, Advantage ${ }^{\circledR}$ Multi (Elanco, Greenfield, IN) and pyrantel

188 pamoate, Nemex-2® (Zoetis, Kalamazoo, MI). In some instances, products labelled for

189 large animals were used; these included moxidectin, Quest ${ }^{\circledR}$ Plus (Zoetis, Kalamazoo, MI)

190 and albendazole, Valbazen ${ }^{\circledR}$ (Zoetis, Kalamazoo, MI). These products were administered

191 orally at $3.3 \mathrm{mg} / \mathrm{kg}$ and $19 \mathrm{mg} / \mathrm{kg}$, respectively. In some cases, compounded drugs were

192 used, such as the PPM Triwormer (Roadrunner pharmacy, Phoenix, AZ).

\subsection{Ancylostoma caninum isotype $\square 1$ beta $\square$ tubulin deep amplicon sequencing}

\subsubsection{DNA preparation}

After setting up the in vitro assays, the remaining eggs were transferred to $2 \mathrm{ml}$

196 cryotubes (Sigma-Aldrich, St. Louis, MO), suspended in a final concentration of $70 \%$

197 ETOH and stored at $-80^{\circ} \mathrm{C}$ until further use. DNA lysates were prepared from individual or

198 pooled egg samples. Briefly, 3 freeze-thaw cycles were carried out at $-80^{\circ} \mathrm{C}$ and at $55^{\circ} \mathrm{C}$

199 respectively, followed by adding $180 \mu \mathrm{L}$ of DirectPCR (Cell) Lysis Buffer (Catalog No.

200 301-C, Viagen Biotech, St. Louis, MO) and $20 \mu \mathrm{L}$ of Proteinase K (Catalog No. 19133,

201 QIAGEN, Hilden, Germany). Samples were then incubated for at least $12 \mathrm{~h}$ at $65^{\circ} \mathrm{C}$, then $1 \mathrm{~h}$

202 at $95^{\circ} \mathrm{C}$, and were then cooled to $4^{\circ} \mathrm{C}$. DNA was purified from the crude DNA lysates using

203 the QIAGEN QIAmp DNA mini kit (Cat\# 51306), following the manufacturer's 204 recommended protocol, and stored at $-80^{\circ} \mathrm{C}$. 
207 synonymous single nucleotide polymorphisms (SNP) at codons 167, 198 and 200 of the $A$.

208 caninum isotype-1 $\beta$-tubulin gene were applied to 70 samples ranging from $200-20,000$

209 eggs with an average of 978 eggs from the two adoption kennels, one active racing kennel,

210 and from one of the veterinary practices. Using adapted primers suitable for Illumina next-

211 generation sequencing, two separate regions of the A. caninum isotype-1 $\beta$-tubulin gene,

212 comprising 293 bp and 340 bp which encompass codon 167, and codons 198 and 200,

213 respectively, were PCR amplified (Jimenez Castro et al., 2019). The following PCR

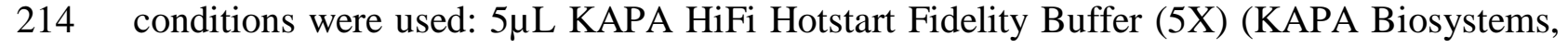

215 USA), $1.25 \mu \mathrm{L}$ Forward Primer $(10 \mu \mathrm{M}), 1.25 \mu \mathrm{L}$ Reverse Primer $(10 \mu \mathrm{M}), 0.75 \mu \mathrm{L}$ dNTPs

$216(10 \mu \mathrm{M}), 0.5 \mu \mathrm{L}$ KAPA HiFi Polymerase (0.5U), 0.1 $\mu \mathrm{L}$ Bovine Serum Albumin (Thermo

217 Fisher Scientific), $14.15 \mu \mathrm{L} \mathrm{H} 2 \mathrm{O}$, and $2 \mu \mathrm{L}$ of DNA lysate. The thermocycling parameters

218 were $95 \square$ for $3 \mathrm{~min}$, followed by 45 cycles of $98 \square$ for $20 \mathrm{~s}, 65 \square$ for $15 \mathrm{~s}$, and $72 \square$ for

21930 s, followed by $72 \square$ for 2 min. Sample purification and addition of barcoded primers

220 followed the protocols defined in (Avramenko et al., 2019). Library preparation was as

221 previously described and library sequencing performed using the Illumina MiSeq platform

222 with the $2 \times 300 v 3$ Reagent Kit (Illumina Inc., San Diego, CA, USA) (Avramenko et al.,

223 2015). For the fragment encompassing codon 167, two independent PCR reactions were

224 performed on all 70 samples and the libraries were sequenced in two independent

225 sequencing runs using the Illumina MiSeq platform with the 2 x 300 v3 Reagent Kit.

$226 \quad$ 2.4.3 Sequence analysis

227 Cutadapt v3.2 (Martin, 2011) was used to remove the A. caninum forward and 228 reverse primer sequences. Following adapter trimming, all the forward and reverse reads 229 were processed using the DADA2 bioinformatic pipeline to obtain Amplicon Sequence 
230 Variants (ASVs) (Callahan et al., 2016). During the quality filtering step of the DADA2

231 pipeline, the default setting with the following additional settings were used: (i) forward

232 and reverse reads were trimmed to a length of $280 \mathrm{bp}$ and $190 \mathrm{bp}$, respectively and (ii)

233 reads shorter than 50 bp or with an expected error of $>1$ or $>2$ in the forward and reverse

234 reads respectively were removed. The DADA2 algorithm was then applied to the filtered

235 and trimmed reads to identify the ASVs. Following this, the overlapping forward and

236 reverse reads were merged, allowing a maximum mismatch of $4 \mathrm{bp}$ in the overlap region.

237 The ASVs generated using the DADA2 pipeline were then aligned to the A. caninum

238 isotype-1 $\beta$-tubulin reference sequence (Genbank Accession: DQ459314.1) using a global

239 (Needleman-Wunsch) pairwise alignment algorithm without end gap penalties. Following

240 alignment, the ASVs were discarded if they were $<180 \mathrm{bp}$ or $>350 \mathrm{bp}$ long, or if they had a

241 percentage identity $<70 \%$ to the reference sequence, or if the ASVs had fewer than 200

242 reads in a sample, or if they were not present in two or more samples. This additional

243 filtering ensures the removal of spurious sequences. The codons 167, 198 and 200 were

244 then analyzed for the presence of any variants resulting in non-synonymous changes. The

245 MUSCLE alignment tool was used to align the filtered ASVs from both the fragments with

246 isotype-1 and $2 \beta$-tubulins from other nematodes present in Clade $\mathrm{V}$ of the nematode

247 phylogeny. Using the Geneious tree builder, a neighbour-joining tree, utilizing the Jukes

248 Cantor tree building method, was constructed from the trimmed alignment, and having $H$.

249 contortus isotype-3 $\beta$-tubulin as the outgroup (Genbank Accession: HE604101) and 2000

250 bootstrap replicates.

\subsection{Data analyses}

Dogs treated solely with albendazole and moxidectin were represented in each of 253 the post-treatment timeframe categories, therefore for each drug individually, statistical 
254 analyses were performed to determine if the FEC of the dogs differed between those

255 categories. For this a Kruswal-Wallis test was performed for the overall comparison with a

256 Benjamini-Krieger-Yekutielli procedure for individual pairwise comparisons. For the EHA

257 and LDA, dose-response analyses were performed after log transformation of the drug

258 concentrations and constraining the bottom value to zero. The top parameter for samples

259 that did not reach $100 \%$ inhibition was constrained to 100 to avoid introducing artificial

260 bias to the model. Data were then fitted to a four-parameter non-linear regression algorithm

261 with variable slope. The $\mathrm{IC}_{50}$ or $\mathrm{IC}_{95}$ values, which represent the concentration of drug

262 required to inhibit hatching (EHA) or development to the third larval stage (LDA) by $50 \%$

263 or $95 \%$ of the maximal response were calculated.

264 The software https://epitools.ausvet.com.au/ was used to calculate the prevalence

265 along with 95\% confidence intervals. For comparing the infection prevalence between

266 states, a Chi-square test was performed for the overall comparison, followed by a Fisher's

267 exact test with a Bonferroni procedure for individual pairwise comparisons between states.

268 All analyses were designed to maintain the overall type I error rate at 5\%. To quantify the

269 relationship between the EHA and the deep-amplicon sequencing assay to measure levels

270 of BZ resistance, a Spearman correlation analysis was performed comparing both $\mathrm{IC}_{50}$ and

$271 \mathrm{IC}_{95}$ values with the F167Y SNP frequencies. All statistical analyses were performed in

272 GraphPad Prism® version 9.0.2, GraphPad Software, San Diego, CA, USA.

\section{Results}

\subsection{Fecal egg count data}

171 of the 219 fecal samples from racing or recently retired greyhounds were

276 positive for hookworm eggs, yielding an overall prevalence of 79\%, with a mean FEC of 
278 Sanford, FL $(p<0.0001)$ kennels were significantly higher than for the Dallas, TX kennel.

279 Percent reductions in FEC following treatments could not be calculated since pre-treatment 280 FEC were not available. However, in the $125(57.3 \%)$ samples collected 2 - 21 days post281 treatment, the mean FEC was 721 EPG, indicating a major lack of efficacy across the 282 different treatments. For the group of samples collected following moxidectin treatment, 283 samples collected $>21$ days post-treatment had a statistically significant higher mean FEC 284 when compared to the other two categories. For the group of samples collected following 285 albendazole treatment, the 10-21 and $>21$ days categories had a statistically significant 286 higher mean FEC when compared to the <10 days. Interestingly, a single group of samples 287 from nine dogs recently acquired from a breeding farm in KS had <5 EPG following 288 treatment. For all other sites, mean FEC were 330 EPG or greater (Table 2).

\subsection{In vitro assays}

EHA and LDA were performed on 35 samples, yielding dose-response data on 23

292 and 22 samples, respectively, which represented samples collected from 81 greyhounds 293 (Fig. 1).

294 Mean and median $\mathrm{IC}_{50}$ and $\mathrm{IC}_{95}$ values were $5.3 \mathrm{uM}, 3.6 \mathrm{uM}$, and 24.5 uM, $23.4 \mathrm{uM}$ for the 295 EHA, respectively. For the LDA, mean and median $\mathrm{IC}_{50}$ values were $749.8 \mathrm{nM},>1000 \mathrm{nM}$, 296 respectively (Table 3). $\mathrm{IC}_{95}$ values were calculated for the LDA (Table 3), but since the $297 \mathrm{IC}_{50}$ were greater than the highest concentration tested in the majority of samples, the mean 298 and median values for $\mathrm{IC}_{95}$ have no real usefulness and are not reported. 
The three codons in the isotype-1 $\beta$-tubulin gene known to have BZ resistance-

302 associated polymorphisms (167, 198 and 200) in Strongylid nematodes were examined

303 using deep-amplicon sequencing. The average read depth for the fragment containing

304 codon 167 was $\sim 10,400$, ranging between 3,180 to 24,732 reads across the samples. For the

305 fragment containing codons 198 and 200, the average read depth was $\sim 22,700$, ranging

306 between 7,890 to 37,060 reads. Only the F167Y (TTC>TAC) resistance polymorphism was

307 detected and this was present in 99\% (69/70) of the samples that were sequenced, and this

308 polymorphism was found at high frequencies in most positive samples (Fig. 2). In 48 out of

309 the 70 samples $(69 \%)$, the frequency of the resistant allele was $\geq 75 \%$. In $29 \%$ of the

310 samples, and in at least one sample from each greyhound kennel, the allele frequency was

$311 \geq 90 \%$. All greyhound kennels had samples with at least a $60 \%$ frequency of the F167Y

312 SNP. Only $7 \%$ of the samples had $<25 \%$ of the resistant allele, and only one sample had

$313100 \%$ frequency of the susceptible allele. These frequencies were consistent between the

314 two independent sequencing runs for the fragment containing codon 167. The ASVs for the

315 amplicons spanning codons 167 and codons 198 and 200 were aligned to other nematode $\beta$ -

316 tubulins using MUSCLE alignment tool. This alignment was trimmed, and a neighbour-

317 joining tree was constructed using Geneious tree builder with $H$. contortus isotype-3 $\beta$ -

318 tubulin (Genbank accession: HE604101). All the ASVs formed a monophyletic cluster with

319 Ancylostoma isotype-1 $\beta$-tubulin (supplementary figure 1 and 2).

$320 \quad 3.4$ Comparison of F167Y (TTC>TAC) frequency and EHA phenotype

$321 \quad$ Both EHA (phenotypic) and $\beta$-tubulin allele (genotypic) data were only available

322 for 15 samples. There was not a statistically significant correlation between the $\mathrm{IC}_{50}$ and 
323 resistant SNP F167Y allele frequency $(p=0.08)$, however, there was a significant

324 correlation with the $\mathrm{IC}_{95}(p=0.04)($ Fig. 4).

\section{Discussion and conclusions}

The present study provides strong and conclusive evidence that racing greyhounds

327 in the USA are infected with MDR A. caninum at a very high prevalence, and with wide

328 geographic distribution. Very high $\mathrm{IC}_{50}$ and $\mathrm{IC}_{95}$ values were measured for both the

329 benzimidazoles and macrocyclic lactones when compared to the susceptible isolate from

330 our previous work (Jimenez Castro et al., 2019), indicating that almost every sample was

331 resistant to both drugs. The F167Y (TTC>TAC) benzimidazole resistance polymorphism

332 was detected, in $99 \%$ of the samples, and at high frequencies in more than $2 / 3$ of the

333 samples. All three greyhound kennels had samples with at least a $60 \%$ frequency of the

334 F167Y SNP, and every sample from the Sanford, FL site had at least a 70\% frequency of

335 the resistant SNP. These data are consistent with levels we reported for our MDR (Worthy)

336 lab isolate, which yielded F167Y SNP frequencies of $87.6-94.5 \%$ over several different

337 passages (Jimenez Castro et al., 2019). Furthermore, in a controlled efficacy study using

338 Worthy $4.1 \mathrm{~F} 3 \mathrm{P}$, we measured an efficacy of $26 \%$ for fenbendazole, confirming that this

339 high F167Y SNP frequency was associated with a very low in vivo efficacy (Jimenez

340 Castro et al., 2020).

341 Although additional genetic analyses are required for confirmation, the available

342 clinical and genetic evidence strongly suggests that these MDR A. caninum evolved on

343 greyhound breeding farms and kennels. Thus, it is germane to this issue to examine the

344 clinical and epidemiological factors that may be responsible for the development of these

345 MDR worms, and to hypothesize why this problem became so severe before it was 346 recognized. Ancylostoma caninum is the most prevalent parasitic nematode in racing 
347 greyhounds (Ash et al., 2019; Jacobs and Prole, 1976), and this is attributed to the near

348 constant exposure of these dogs to infective third stage larvae in the sand/dirt exercise

349 run/pens, which are ideal for hookworm transmission (Ridley et al., 1994). The

350 combination of large numbers of animals and high transmission rates produces large

351 effective populations of worms and increases the probabilities that resistance mutations will

352 occur (Gilleard, 2006; Redman et al., 2015). Racing greyhounds are also treated extremely

353 frequently with multiple different anthelmintics (e.g., fenbendazole, ivermectin, pyrantel)

354 throughout their lives (Ridley et al., 1994). The intervals between these treatments often are

355 less than the pre-patent period for hookworms, which will minimize the amount of refugia.

356 Thus, genetically-resistant worms surviving treatment will have a large reproductive

357 advantage, and the lack of refugia will lead to a rapid increase in their frequency (Martin et

358 al., 1981; van Wyk, 2001). This combination of factors is known to place heavy selection

359 pressure for drug resistance in nematodes (Wolstenholme et al., 2004), and is very similar

360 to the epidemiological factors that have led to high levels of MDR in nematodes of sheep

361 and goats, worldwide (Kaplan and Vidyashankar, 2012).

362 Given the fact that resistance is likely to have developed independently to each drug

363 class, and the fact that MDR A. caninum to all three major anthelmintic classes seem to be

364 virtually ubiquitous in racing greyhounds in the USA, one must ask the question; "why has

365 resistance to any single drug class not been reported previously in the USA greyhound

366 population"? To answer this question, we must examine how veterinarians typically

367 manage hookworm infections in dogs. Typically, when a dog presents to a veterinarian with

368 a fecal positive for hookworms, the dog is treated with one or more drugs from the

369 benzimidazole, macrocyclic lactone or tetrahydropyrimidine classes. If the dog then tests

370 positive again in a future exam, the infection is attributed to reinfection or reactivation of 
371 encysted/arrested larvae (larval leak). Consequently, the same treatment regimen is often

372 repeated, or the veterinarian may choose to use a drug from a different drug class. One

373 thing that was never done by small animal clinicians in the past, is measuring the efficacy

374 of the treatment in a fecal egg count reduction test (FECRT) (Jimenez Castro and Kaplan,

375 2020; Kaplan, 2020), by performing both pre- and post-treatment FEC. As a result,

376 anthelmintic resistance (AR) is not diagnosed, and most often is not even considered as a

377 likely cause of the recurrent hookworm infections. Therefore, as resistance evolves and

378 leads to more recurrent hookworm infections, veterinarians typically treat more often, and

379 rotate and/or combine drugs. But they do not perform FECRT to measure the efficacy of

380 the various drugs administered. Thus, as long as one drug remains efficacious, the problem

381 will appear to be managed, and recurrent infections will continue to be attributed to

382 reinfection or reactivation of encysted/arrested larvae. However, once MDR to all drugs

383 evolves, it is no longer possible to manage the infections, and the problem of anthelmintic

384 resistance becomes more obvious. Our data demonstrate evidence of very high $\mathrm{IC}_{50}$ and

$385 \mathrm{IC}_{95}$ values for benzimidazoles and the macrocyclic lactones. With regards to pyrantel, no

386 in vitro or molecular assays currently exist for measuring resistance. However, in every

387 suspected MDR case we have treated with pyrantel pamoate there is virtually no efficacy

388 based on FEC reduction, (Jimenez Castro et al., 2019), and in a controlled efficacy study

389 using Worthy 4.1F3P, pyrantel pamoate yielded an efficacy of only 23\% (Jimenez Castro et

390 al., 2020).

391 Regarding benzimidazole resistance, the Sanford, FL kennel applied the greatest

392 benzimidazole selection pressure of any of the kennels, treating all dogs twice a month with

393 albendazole, and all dogs tested had F167Y (TTC>TAC) frequencies of at least $70 \%$.

394 When comparing the phenotypic and genotypic data for benzimidazole, the $\mathrm{IC}_{95}$ yielded a 
significant correlation $(p=0.04)$ but the $\mathrm{IC}_{50}$ did not $(p=0.08)$. The lack of significance for

396 the $\mathrm{IC}_{50}$ may be due to low power, as a consequence of only having 15 samples with both

397 types of data. However, this finding is consistent with our previous work where we found

398 that the $\mathrm{IC}_{95}$ was more appropriate for discriminating susceptible vs resistant isolates using

399 the EHA (Jimenez Castro et al., 2019). Interestingly, there were two samples, D1 13 and D1

40014 that had $\mathrm{IC}_{95}$ values 13 and 18 times higher than the susceptible isolate from our

401 previous work (Jimenez Castro et al., 2019), but had a resistant F167Y SNP allele

402 frequency of only 5\%, and $0 \%$, respectively. This lack of correlation between phenotype

403 and genotype raises three possible explanations: (1) the EHA has a high interassay

404 variability, (2) there are mutations, other than at codons 167, 198 and 200, that are involved

405 with resistance to benzimidazole drugs, or (3) there are loci other than $\beta$-tubulin that are

406 involved with resistance to benzimidazole drugs. However, in on our previous work we

407 tested multiple isolates and biological replicates and had rather low interassay variability

408 (Jimenez Castro et al., 2019). Additionally, we previously measured a >100-fold increase in

409 the EHA $\mathrm{IC}_{50}$ in the Worthy isolate following treatment with fenbendazole, but the SNP

410 allele frequency remained relatively unchanged (Jimenez Castro et al., 2019). Together,

411 these findings lend support to the hypothesis that there are non- $\beta$-tubulin mutations that are

412 involved in resistance to benzimidazole drugs. Evidence of this has already been reported in

413 Caenorhabditis spp. where a quantitative trait loci that did not overlap with $\beta$-tubulin genes

414 was identified in two genetically divergent isolates (Zamanian et al., 2018). Also, using

415 genome wide association mappings in C. elegans, novel genomic regions independent of

416 ben-1 and other $\beta$-tubulin loci were correlated with resistance to albendazole (Hahnel et al.,

417 2018). Additionally, disparity in responses in C. elegans to fenbendazole and albendazole

418 showed evidence that the former could have additional targets beyond $\beta$-tubulin, such as 
419 genes that encode $\beta$-tubulin interacting proteins (Dilks et al., 2020). Thus, our observations 420 demand further study.

421 Our previous work demonstrated that the LDA provided excellent discrimination 422 between susceptible and resistant isolates for the MLs (Jimenez Castro et al., 2019). In the 423 current study the median $\mathrm{IC}_{50}$ value was $>83$ times higher than the susceptible isolate value 424 from our previous work. This is both extremely high and an underestimation, since the $\mathrm{IC}_{50}$ 425 of many samples could not be accurately measured given that they exceeded the highest 426 concentration tested. Furthermore, of all the samples that had LDA assays with $\mathrm{IC}_{50}$ values $427>1000 \mathrm{nM}$, all but one had moxidectin as the last treatment administered, and that one 428 group was administered moxidectin in the previous treatment. Macrocyclic lactones, 429 particularly, ivermectin, have been used intensively by the greyhound industry for parasite 430 control for the past several decades (Ridley et al., 1994). However, to our knowledge, 431 moxidectin, which is a substantially more potent member of this drug class (Prichard et al., 432 2012), has only recently started to be used on greyhound farms and kennels. In $H$. 433 contortus, ivermectin resistant worms that are naïve to moxidectin are typically killed at 434 very high efficacy following administration of moxidectin (Craig et al., 1992; Oosthuizen 435 and Erasmus, 1993); however, once moxidectin is used regularly in an ivermectin-resistant 436 population, resistance to moxidectin can develop rapidly (Kaplan et al., 2007). Evidence 437 suggests this same pattern is occurring in hookworms as well.

438 When examining the FEC data for moxidectin and albendazole by timeframe post439 treatment, we found significant differences for both drugs, but in a different pattern. For the 440 group of samples collected following albendazole treatment, the 10-21 $(p=0.0003)$ and $441>21$ days $(p=0.0004)$ categories had a statistically significant higher mean FEC when 442 compared to the $<10$ days category. This finding is consistent with our previous 
443 observations in the Worthy isolate, where we documented a temporary suppression on

444 worm fecundity following treatment with fenbendazole (Jimenez Castro et al., 2019;

445 Jimenez Castro et al., 2020). In contrast, for the dogs treated with moxidectin, there was no

446 difference between the $<10$ and 10-21 day timeframes, but the $>21$ day period had

447 significantly higher FEC $(p=0.0002)$ and $(p<0.0001)$, respectively. This is also consistent

448 with our previous observations where no reduction in fecundity was seen in moxidectin-

449 resistant $A$. caninum following treatment with ML drugs. The higher EPG in the >21day

450 period is likely due to reinfection and/or reactivation of arrested larvae.

451 The almost ubiquitous presence of MDR worms in recently retired greyhounds, 452 combined with the demise of the greyhound racing industry and increasing numbers of 453 greyhound adoptions, poses a serious risk to the health of pet dogs. From 2009-2019, the 454 number of dog parks increased by $74 \%$ in the USA (TPL, 2019), and a recent survey 455 showed that the prevalence of $A$. caninum in dogs visiting these parks (Stafford et al., 2020) 456 was more than $70 \%$ higher as compared to the prevalence in all pet dogs recorded over the 457 same general timeframe (Sweet et al., 2021). These relative prevalence data should not be 458 surprising; a fecal pile deposited by a $30 \mathrm{~kg}$ dog with an A. caninum FEC of $\sim 1000$ EPG 459 will contain approximately 500,000 eggs. If not picked up, tens to hundreds of thousands of 460 infective larvae are likely to contaminate the surrounding soil from this one defecation. 461 Consequently, there is a high probability of transmission to other dogs visiting the dog 462 park, and once infected, usual anthelmintic treatment of these dogs will have little efficacy. 463 The end result will be a continual cycle of infection and transmission that is not interrupted 464 by usual monthly treatments with heartworm preventive products, or with anthelmintics 465 administered specifically to treat the hookworm infections. When this is considered in light 466 of the fact that resistance in A. caninum was not reported in greyhounds until the worms 
467 were already MDR, it seems highly likely that anthelmintic-resistant $A$. caninum are 468 already quite common in pet dogs.

469 Finally, given these new alarming data, it is urgent that studies be performed to 470 determine the prevalence and geographic distribution of drug-resistant A. caninum in the 471 general pet dog population. Additionally, studies investigating the haplotype diversity of 472 the susceptible and resistant alleles in A. caninum isolates from both greyhounds and the 473 general pet dog population from different geographic regions are likely to provide deeper 474 insights into the molecular epidemiology and the origin(s) of these MDR worms.

\section{Acknowledgements}

We thank the staff at the participating greyhound kennels, adoption groups and veterinarians for their efforts and assistance, which made this project possible.

\section{Conflict of interest statement}

480 The authors do not report any conflict of interests.

\section{References}

Ash, A., Lymbery, A., Godfrey, S., Shiel, R., Paul, A., 2019. Substrate type and age are risk factors for gastrointestinal parasitism in greyhound kennels. Vet Parasitol 265, 7-14. Avramenko, R.W., Redman, E.M., Lewis, R., Yazwinski, T.A., Wasmuth, J.D., Gilleard, J.S., 2015. Exploring the Gastrointestinal "Nemabiome": Deep Amplicon Sequencing to Quantify the Species Composition of Parasitic Nematode Communities. PLoS One 10, 1-18. Avramenko, R.W., Redman, E.M., Melville, L., Bartley, Y., Wit, J., Queiroz, C., Bartley, D.J., Gilleard, J.S., 2019. Deep amplicon sequencing as a powerful new tool to screen for sequence polymorphisms associated with anthelmintic resistance in parasitic nematode populations. Int J High-resolution sample inference from Illumina amplicon data. Nature Methods 13, 581-583. ivermectin-resistant strain of Haemonchus contortus in sheep. Vet Parasitol 41, 329-333. 
Dilks, C.M., Hahnel, S.R., Sheng, Q., Long, L., McGrath, P.T., Andersen, E.C., 2020. Quantitative benzimidazole resistance and fitness effects of parasitic nematode beta-tubulin alleles. International Journal for Parasitology: Drugs and Drug Resistance 14, 28-36. Drake, J., Carey, T., 2019. Seasonality and changing prevalence of common canine gastrointestinal nematodes in the USA. Parasites \& vectors 12, 430. Epe, C., 2009. Intestinal nematodes: biology and control. Vet Clin North Am Small Anim Pract 39, 1091-1107, vi-vii. F.D.A, 1983. NADA 121-473 Panacur, Food and Drug Administration. F.D.A, 1993. NADA 141-008 Drontal, Food and Drug Administration. F.D.A, 1994. NADA 141-007 Drontal Plus, Food and Drug Administration. F.D.A, 1998. NADA 140-915 Interceptor, Food and Drug Administration. F.D.A, 2006. NADA 141-251 Advantage Multi, Food and Drug Administration. Gartland, J., 2021. National Greyhound Association. Gilleard, J.S., 2006. Understanding anthelmintic resistance: the need for genomics and genetics. Int J Parasitol 36. allelic heterogeneity at a Caenorhabditis elegans beta-tubulin locus explains natural resistance to benzimidazoles. PLOS Pathogens 14, 1-26. Hall, A.P., 2016. The Economics of Restoring Live Horse Racing and Greyhound Racing in Kansas, in: Alliance, T.G.K.R. (Ed.), Kansas University School of Business. Hotez, P.J., Brooker, S., Bethony, J., Bottazzi, M.E., Loukas, A., Xiao, S., 2004. Hookworm infection. New England Journal of Medicine 351, 799-807. Jacobs, D., Prole, J., 1976. Helminth infections of British dogs: prevalence in racing greyhounds. Veterinary parasitology 1, 377-387. Jimenez Castro, P.D., Howell, S.B., Schaefer, J.J., Avramenko, R.W., Gilleard, J.S., Kaplan, R.M., 2019. Multiple drug resistance in the canine hookworm Ancylostoma caninum: an emerging threat? Parasite \& Vectors 12, 576.

Jimenez Castro, P.D., Kaplan, R.M., 2020. Persistent or suspected-resistant hookworm infections, Clinician's Brief, pp. 59-68.

Jimenez Castro, P.D., Mansour, A., Charles, S., Hostetler, J., Settje, T., Kulke, D., Kaplan, R.M., 2020. Efficacy evaluation of anthelmintic products against an infection with the canine hookworm (Ancylostoma caninum) isolate Worthy 4.1F3P in dogs. International Journal for Parasitology: Drugs and Drug Resistance 13, 22-27.

Kalkofen, U.P., 1987. Hookworms of dogs and cats. Vet Clin North Am Small Anim Pract 17, 13411354.

Kaplan, R.M., 2020. Biology, Epidemiology, Diagnosis, and Management of Anthelmintic Resistance in Gastrointestinal Nematodes of Livestock. Veterinary Clinics of North America: Food Animal Practice 36, 17-30.

Kaplan, R.M., Vidyashankar, A.N., 2012. An inconvenient truth: Global worming and anthelmintic resistance. Veterinary Parasitology 186, 70-78.

Kaplan, R.M., Vidyashankar, A.N., Howell, S.B., Neiss, J.M., Williamson, L.H., Terrill, T.H., 2007. A novel approach for combining the use of in vitro and in vivo data to measure and detect emerging moxidectin resistance in gastrointestinal nematodes of goats. Int J Parasitol 37, 795-804.

Lima, V.F.S., Cringoli, G., Rinaldi, L., Monteiro, M.F.M., Calado, A.M.C., Ramos, R.A.N., MeiraSantos, P.O., Alves, L.C.J.P.R., 2015. A comparison of mini-FLOTAC and FLOTAC with classic 541 methods to diagnosing intestinal parasites of dogs from Brazil. Parasitology Research 114, 35295423533. 
Little, S.E., Johnson, E.M., Lewis, D., Jaklitsch, R.P., Payton, M.E., Blagburn, B.L., Bowman, D.D., Moroff, S., Tams, T., Rich, L., Aucoin, D., 2009. Prevalence of intestinal parasites in pet dogs in the United States. Vet Parasitol 166, 144-152.

Lord, L.K., Yaissle, J.E., Marin, L., Couto, C.G., 2007. Results of a web-based health survey of retired racing Greyhounds. Journal of veterinary internal medicine 21, 1243-1250. Martin, M., 2011. Cutadapt removes adapter sequences from high-throughput sequencing reads. EMBnet. journal 17, 10-12.

Martin, P.J., Le Jambre, L.F., Claxton, J.H., 1981. The impact of refugia on the development of thiabendazole resistance in Haemonchus contortus. International Journal For Parasitology 11, 3541.

Maurelli, M.P., Rinaldi, L., Alfano, S., Pepe, P., Coles, G.C., Cringoli, G., 2014. Mini-FLOTAC, a new tool for copromicroscopic diagnosis of common intestinal nematodes in dogs. Parasite \& Vectors 7, 356.

Oosthuizen, W.T., Erasmus, J.B., 1993. Efficacy of moxidectin against a strain of Haemonchus contortus resistant to ivermectin, a benzimidazole and a salicylanilide. Journal of the South African Veterinary Association 64, 9-12. Prichard, R., Ménez, C., Lespine, A., 2012. Moxidectin and the avermectins: Consanguinity but not identity. International Journal for Parasitology: Drugs and Drug Resistance 2, 134-153. Redman, E., Whitelaw, F., Tait, A., Burgess, C., Bartley, Y., Skuce, P.J., Jackson, F., Gilleard, J.S., 2015. The emergence of resistance to the benzimidazole anthlemintics in parasitic nematodes of livestock is characterised by multiple independent hard and soft selective sweeps. PLoS Negl Trop Dis 9, e0003494.

Ridley, R.K., Dryden, M.W., Gabbert, N.H., Schoning, P., 1994. Epidemiology and Control of Helminth-Parasites in Greyhound Breeding Farms. Comp Cont Educ Pract 16, 585-596. Stafford, K., Kollasch, T.M., Duncan, K.T., Horr, S., Goddu, T., Heinz-Loomer, C., Rumschlag, A.J., Ryan, W.G., Sweet, S., Little, S.E., 2020. Detection of gastrointestinal parasitism at recreational canine sites in the USA: the DOGPARCS study. Parasites \& vectors 13, 275.

Stassens, P., Bergum, P.W., Gansemans, Y., Jespers, L., Laroche, Y., Huang, S., Maki, S., Messens, J., Lauwereys, M., Cappello, M., Hotez, P.J., Lasters, I., Vlasuk, G.P., 1996. Anticoagulant repertoire of the hookworm Ancylostoma caninum. Proceedings of the National Academy of Sciences 93, 21492154. State, F.D.O., 2018. Results of elections.

Sweet, S., Hegarty, E., Mccrann, D.J., Coyne, M., Kincaid, D., Szlosek, D., 2021. A 3-year retrospective analysis of canine intestinal parasites: fecal testing positivity by age, U.S. geographical region and reason for veterinary visit. Parasites \& vectors 14.

Taylor, M., Coop, R.L., Wall, R., 2016. Veterinary Parasitology. Wiley Blackwell.

Theil, C.M., 2021. GREY2K USA

TPL, 2019. Dog park rankings for the 100 largest U.S. cities.

van Wyk, J.A., 2001. Refugia--overlooked as perhaps the most potent factor concerning the development of anthelmintic resistance. The Onderstepoort journal of veterinary research $68,55-$ 67.

Wolstenholme, A.J., Fairweather, I., Prichard, R., Samson-Himmelstjerna, G., Sangster, N.C., 2004. Drug resistance in veterinary helminths. Trends in parasitology 20.

586 Zamanian, M., Cook, D.E., Zdraljevic, S., Brady, S.C., Lee, D., Lee, J., Andersen, E.C., 2018. Discovery 587 of genomic intervals that underlie nematode responses to benzimidazoles. PLOS Neglected 588 Tropical Diseases 12, e0006368. 


\section{FIGURE LEGENDS}

591 Fig. 1. Scatterplots of greyhound samples showing the log transformed Egg Hatch Assay

592 (EHA) $\mathrm{IC}_{50}$ (a) and $\mathrm{IC}_{95}$ (b) values, and the Larval Development Assay (LDA) $\mathrm{IC}_{50}$ (c)

593 values for the benzimidazoles (BZs) and macrocyclic lactones (MLs), respectively. Each

594 dark blue and light blue dot represent an assay performed on an individual or a pooled

595 sample, respectively. The black dot represents the value of our susceptible laboratory

596 isolate for reference. Dose-responses were analysed using the variable slope nonlinear

597 regression model analysis contained in GraphPad 9.0.2.

599 Fig. 2. The relative proportions of isotype-1 beta-tubulin alleles encoding resistance

600 conferring polymorphisms at F167Y vs wild type susceptible as measured by deep-

601 amplicon sequencing in 70 samples from greyhounds that originated from 16 different

602 locations in 8 different states.

603

604 Fig. 3. The relative proportions of isotype-1 beta-tubulin alleles in the fragment containing

605 codons 198 and 200 as measured by deep-amplicon sequencing in 70 samples from

606 greyhounds that originated from 16 different locations in 8 different states.

609 Fig 4. Scatterplots of EHA IC 50 values (A) or IC $_{95}$ values (B) vs. F167Y SNP frequency

610 based on deep-amplicon sequencing. Only 15 samples had results from both assays. The

611 gray dot represents a pooled sample. The highest concentration tested in the EHA was 40

$612 \mathrm{uM}$, therefore the four values with $\mathrm{IC}_{95}$ of $40 \mathrm{uM}$ likely would have been greater if higher

613 concentrations were tested. 
bioRxiv preprint doi: https://doi.org/10.1101/2021.04.24.438405; this version posted April 24, 2021. The copyright holder for this preprint (which was not certified by peer review) is the author/funder. All rights reserved. No reuse allowed without permission.

\section{4}

615

616 

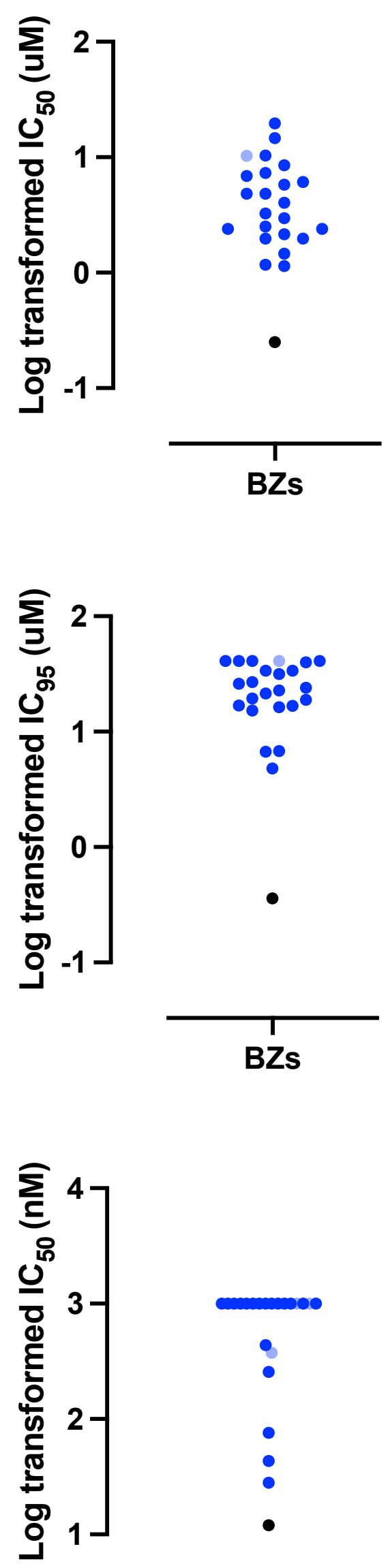

I

MLs 


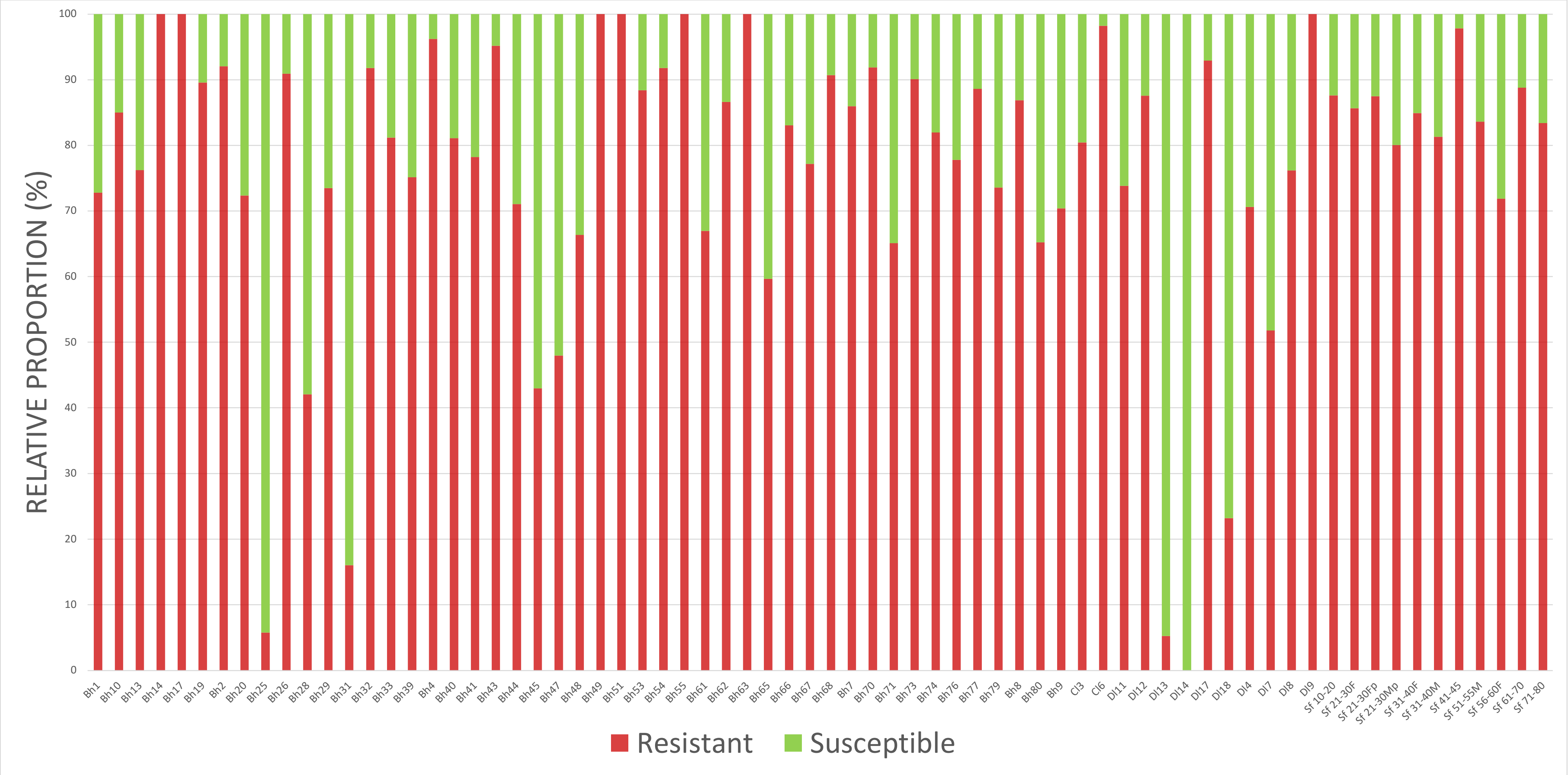




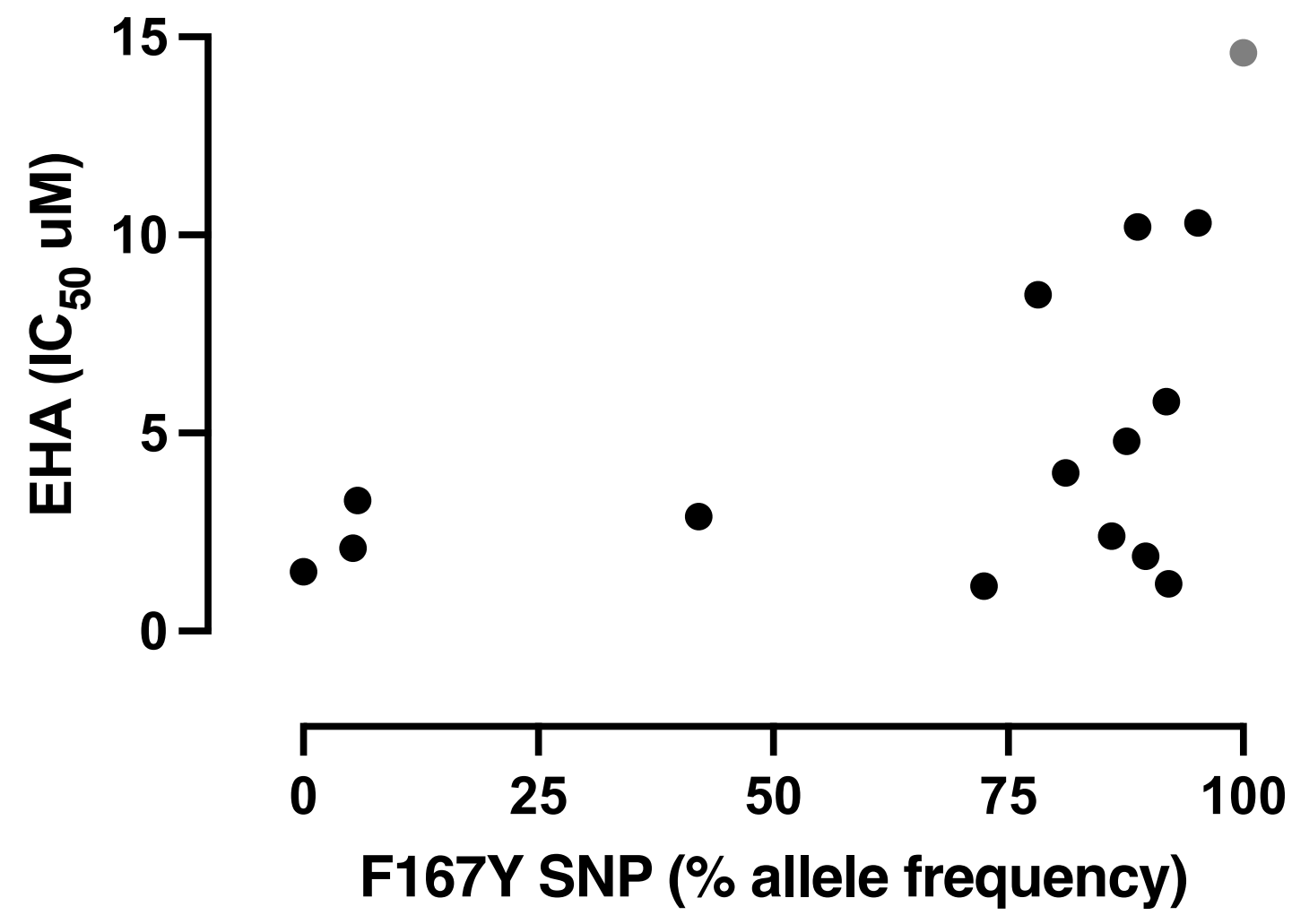

B

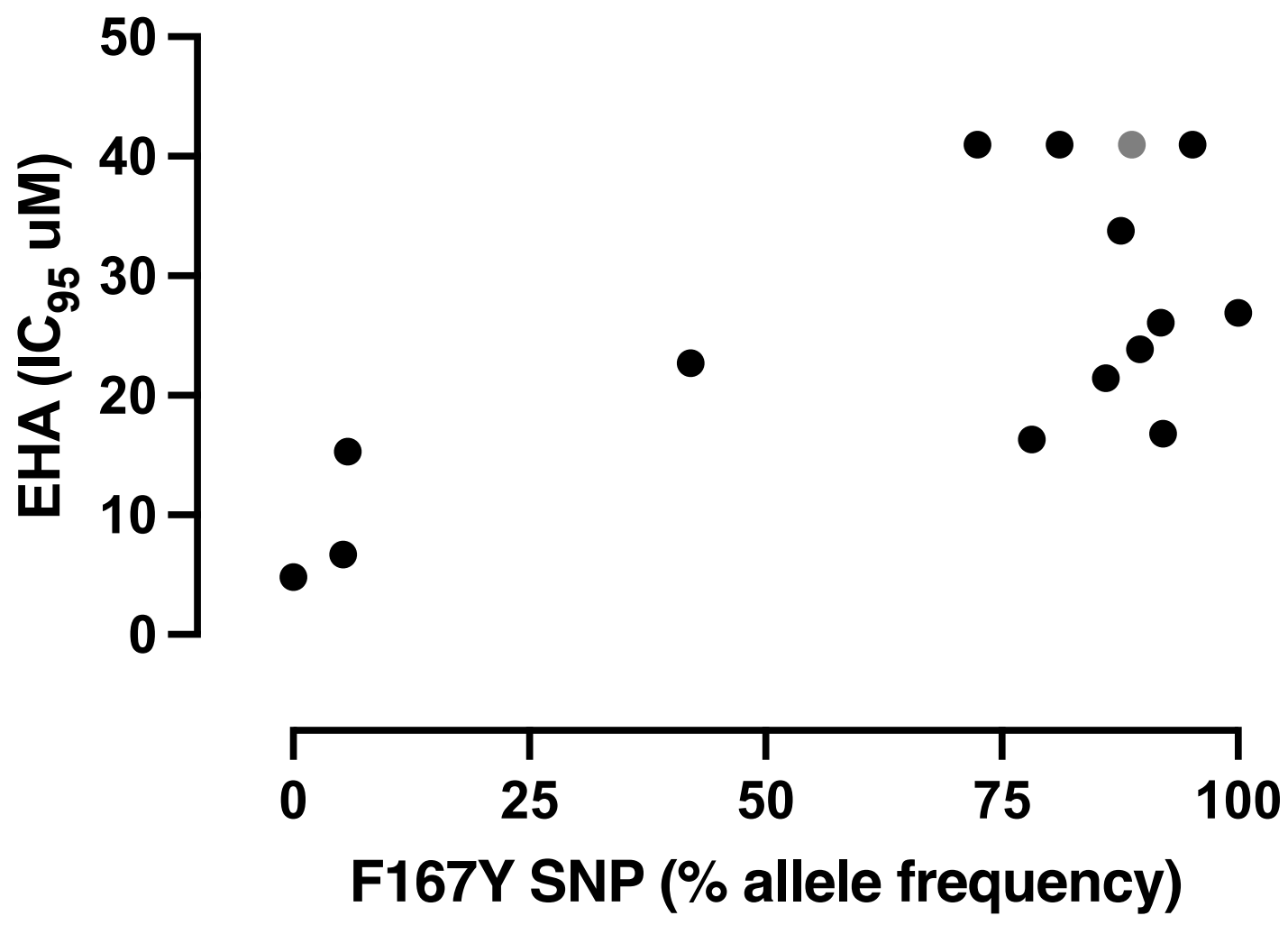


Table 1. Mean fecal egg count (FEC) data and percent prevalence of hookworm infections from 219 greyhound dog samples obtained from two greyhound adoption kennels, one greyhound racing kennel, and veterinary practices. Data from the three veterinary practices were combined for reporting purposes. Positive samples were defined as having a FEC of $>5$ eggs per gram (EPG). The software https://epitools.ausvet.com.au/ was used to calculate the prevalence along with $95 \%$ confidence intervals.

\begin{tabular}{cccc}
\hline Adoption kennel & FEC (EPG) & Prevalence $(\boldsymbol{\%})(\mathbf{9 5} \% \mathbf{C I})^{*}$ & Total \# of samples \\
\hline Birmingham, AL & 527.8 & $76.3(66.2,84.7)^{\mathrm{a}}$ & 80 \\
Sanford, FL & 1288.6 & $87.5(79.1,93.5)^{\mathrm{a}}$ & 80 \\
Dallas, TX & 683 & $48.1(30.1,66.5)^{\mathrm{b}}$ & 27 \\
\hline Veterinary practices $^{\mathbf{1}}$ & 529.3 & $90.6(77.5,97.6)$ & 32 \\
\hline Overall means & 822.4 & $79(73.3,84.0)$ & 219
\end{tabular}

\footnotetext{
1 Three veterinary practices located in Acworth, GA, Columbia, SC, and St. Petersburg, FL
} * Shared superscripts denote non-significant differences 
Table 2. Mean fecal egg counts (FEC) in eggs per gram (EPG) with the standard error of the mean (SEM) of fecal samples from greyhound dogs. Samples were obtained at varying intervals following treatments with several different anthelmintics. State of origin of the dogs is provided, and where more than one city was represented and city was known, a subscript letter indicates the number of cities represented. Timeframes since the previous anthelmintic treatment were categorized as: (A): <10 days, (B): $10-21$ days, and $(\mathrm{C})$ : $>21$ days. Dogs treated solely with albendazole and moxidectin were represented in each of the post-treatment timeframe categories, therefore for each drug individually, statistical analyses were performed to determine if the FEC of the dogs differed between those categories.

\begin{tabular}{|c|c|c|c|c|}
\hline Treatment & $\begin{array}{c}\text { No. of } \\
\text { greyhounds }\end{array}$ & $\begin{array}{c}\text { Categories post- } \\
\text { treatment* }\end{array}$ & $\begin{array}{l}\text { FEC (SEM) } \\
\text { (EPG) }\end{array}$ & State of origin $^{5}$ \\
\hline \multicolumn{5}{|l|}{ Single drugs } \\
\hline Moxidectin $^{1}$ & 20 & $\mathrm{~A}^{\mathrm{a}}$ & $333.3(108.9)$ & $\mathrm{AL}, \mathrm{FL}_{1}, \mathrm{FL}_{2}, \mathrm{FL}_{3}, \mathrm{AR}, \mathrm{TX}, \mathrm{WV}, \mathrm{KS}$ \\
\hline Moxidectin $^{1}$ & 41 & $\mathrm{~B}^{\mathrm{a}}$ & $334.6(93.5)$ & $\mathrm{AL}, \mathrm{AR}, \mathrm{FL}_{1}, \mathrm{FL}_{2}, \mathrm{FL}_{4}, \mathrm{WV}, \mathrm{AR}, \mathrm{CO}, \mathrm{TX}$ \\
\hline Moxidectin $^{1}$ & 53 & $C^{b}$ & $1117(189.4)$ & $\mathrm{AL}, \mathrm{AR}, \mathrm{FL}_{1}, \mathrm{FL}_{3}, \mathrm{FL}_{4}, \mathrm{FL}_{5}, \mathrm{FL}_{6}, \mathrm{WV}$ \\
\hline Pyrantel pamoate ${ }^{2}$ & 3 & $\mathrm{C}$ & $600(263.2)$ & FL, $\mathrm{AL}$ \\
\hline Albendazole ${ }^{3}$ & 20 & $A^{c}$ & $349.3(150)$ & $\mathrm{AR}, \mathrm{FL}$ \\
\hline Albendazole ${ }^{3}$ & 21 & $B^{d}$ & $1874(396.4)$ & $\mathrm{AR}, \mathrm{FL}$ \\
\hline Albendazole $^{3}$ & 10 & $C^{\mathrm{d}}$ & $1819(524.1)$ & FL \\
\hline \multicolumn{5}{|l|}{ Combinations } \\
\hline Febantel-pyrantel-moxidectin ${ }^{4}$ & 20 & A & $834.8(271.9)$ & $\mathrm{AL}, \mathrm{AR}, \mathrm{AR}_{1}, \mathrm{FL}_{1}, \mathrm{FL}_{3}, \mathrm{FL}_{4}, \mathrm{FL}_{7}$ \\
\hline Moxidectin $^{1}+$ pyrantel $^{2}$ & 9 & $\mathrm{C}$ & 0 & $\mathrm{KS}$ \\
\hline $\begin{array}{l}{ }_{1}^{1} \text { Quest }{ }^{\circledR} \text { Plus } \\
{ }^{2} \text { Nemex }{ }^{\circledR}-2 \\
{ }^{3} \text { Valbazen }{ }^{\circledR} \\
{ }^{4} \text { Drontal }{ }^{\circledR} \text { Plus and Advantage }{ }^{\circledR} \\
{ }^{5} \mathrm{FL}_{1}=\text { Palm Beach, FL } 2 \text { = Daytor }\end{array}$ & ti & 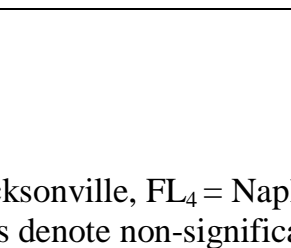 & t. Myers, FL & $=$ Clermont, $\mathrm{FL}_{6}=$ Pensacola, $\mathrm{AR}_{1}=$ West Memphis \\
\hline
\end{tabular}


Table 3. Geographic origin of the dogs, fecal egg counts (FEC) in eggs per gram (EPG), egg hatch assay (EHA) $\mathrm{IC}_{50}$ and $\mathrm{IC}_{95}$ values (uM), larval development assay (LDA) $\mathrm{IC}_{50}$ values and $\mathrm{IC}_{95}$ values $(\mathrm{nM})$, last anthelmintic treatment administered, and days from treatment to sample collection. All dose-response analyses were performed after log transformation of the drug concentrations and constraining the bottom value to zero. Data were then fitted to a four-parameter non-linear regression algorithm with variable slope. 
${ }^{1}$ Samples were received from these locations, greyhound adoption kennels: $\mathrm{Bh}=($ Birmingham, $\mathrm{AL})$, and $\mathrm{Dl}=(\mathrm{Dallas}, \mathrm{TX})$; greyhound racing kennel: Sf = (Sanford, FL), and veterinary practices: $\mathrm{Cl}=($ Columbia, $\mathrm{SC}), \mathrm{Sp}=(\mathrm{St}$. Petersburg, FL), and Ac $=($ Acworth, GA)

${ }^{2}$ Drontal ${ }^{\circledR}$ Plus and Advantage ${ }^{\circledR}$ Multi

${ }^{3}$ Quest $®$ Plus

${ }^{4}$ Valbazen ${ }^{\circledR}$ (albendazole)

${ }^{5}$ PPM Triwormer and Advantage $®$ Multi

${ }^{6}$ Nemex ${ }^{\circledR}-2$

\begin{tabular}{|c|c|c|c|c|c|c|c|c|}
\hline $\begin{array}{l}\text { Sample } \\
\text { ID code }\end{array}$ & $\begin{array}{c}\text { FEC } \\
(\text { EPG) }\end{array}$ & $\begin{array}{l}\text { EHA IC }_{50} \\
\quad(\mathrm{BZ})\end{array}$ & $\begin{array}{l}\text { EHA IC } 95 \\
\quad(B Z)\end{array}$ & $\begin{array}{l}\mathrm{LDA} \mathrm{IC}_{50} \\
\quad(\mathrm{ML})\end{array}$ & $\begin{array}{l}\mathrm{LDA} \mathrm{IC}_{95} \\
\quad(\mathrm{ML})\end{array}$ & Last treatment administered & $\begin{array}{c}\text { Days since } \\
\text { last } \\
\text { treatment }\end{array}$ & $\begin{array}{c}\text { City and State of sample } \\
\text { origin }^{7}\end{array}$ \\
\hline $\mathrm{Bh} 2$ & 4725 & 1.2 & 16.8 & 255.5 & $>1000$ & Febantel-pyrantel-moxidectin ${ }^{2}$ & 4 & Birmingham, AL \\
\hline $\mathrm{Bh} 7$ & 1895 & 2.4 & 21.5 & 43.3 & $>1000$ & Febantel-pyrantel-moxidectin ${ }^{2}$ & 4 & Palmbeach, FL \\
\hline Bh 19 & 1825 & 2 & 24 & 28.1 & $>1000$ & Febantel-pyrantel-moxidectin ${ }^{2}$ & 4 & Palmbeach, FL \\
\hline $\mathrm{Bh} 20$ & 2635 & 1.14 & $>40$ & NA & NA & Febantel-pyrantel-moxidectin ${ }^{2}$ & 4 & Birmingham, AL \\
\hline $\mathrm{Bh} 25$ & 1440 & 3.3 & 15.3 & 75.8 & 315.6 & Moxidectin $^{3}$ & 16 & Birmingham, AL \\
\hline $\mathrm{Bh} 28$ & 1410 & 2.9 & 22.7 & 438.5 & $>1000$ & Moxidectin $^{3}$ & 16 & Birmingham, AL \\
\hline Bh 32 & 520 & 5.8 & 26.1 & $>1000$ & $>1000$ & Moxidectin $^{3}$ & 16 & Birmingham, AL \\
\hline $\mathrm{Bh} 40$ & 690 & 4 & $>40$ & $>1000$ & $>1000$ & Moxidectin $^{3}$ & 16 & Daytona beach, FL \\
\hline $\mathrm{Bh} 41$ & 615 & 8.5 & 16.3 & $>1000$ & $>1000$ & Moxidectin $^{3}$ & 2 & Birmingham, AL \\
\hline Bh 43 & 1675 & 10.3 & $>40$ & $>1000$ & $>1000$ & Moxidectin $^{3}$ & 2 & Birmingham, AL \\
\hline $\mathrm{Bh} 51$ & 205 & 14.6 & 26.9 & $>1000$ & $>1000$ & Moxidectin $^{3}$ & 18 & Birmingham, AL \\
\hline $\mathrm{Bh} 53$ & 2560 & NA & NA & $>1000$ & $>1000$ & Moxidectin $^{3}$ & 11 & Birmingham, AL \\
\hline Sf $10-20 *$ & 472.7 & NA & NA & 375.4 & $>1000$ & Moxidectin $^{3}$ & 31 & Sanford, FL \\
\hline Sf $61-70 *$ & 1353 & 10.2 & $>40$ & $>1000$ & $>1000$ & Moxidectin $^{3}$ & 30 & Sanford, FL \\
\hline Sf $71-80 *$ & 1819 & NA & NA & $>1000$ & $>1000$ & Albendazole $^{4}$ & 30 & Sanford, FL \\
\hline Dl 12 & 4450 & 4.8 & 33.8 & $>1000$ & $>1000$ & Unknown & 25 & $\mathrm{KS}$ \\
\hline Dl 13 & 600 & 2.1 & 6.7 & NA & NA & Unknown & 25 & $\mathrm{KS}$ \\
\hline Dl 14 & 620 & 1.5 & 4.8 & NA & NA & Unknown & 25 & $\mathrm{KS}$ \\
\hline $\mathrm{Cl} 9$ & 1110 & 4.8 & 33.8 & $>1000$ & $>1000$ & Pyrantel-mebendazole-moxidectin ${ }^{5}$ & 4 & Sanford, FL \\
\hline $\mathrm{Cl} 12$ & 490 & NA & NA & $>1000$ & $>1000$ & Pyrantel-mebendazole-moxidectin ${ }^{5}$ & 9 & Sanford, FL \\
\hline $\mathrm{Cl} 14$ & 1180 & 2.4 & 18.9 & $>1000$ & $>1000$ & Moxidectin ${ }^{3}$ & 5 & Birmingham, AL \\
\hline $\mathrm{Cl} 16$ & 1665 & 1.9 & 6.8 & NA & NA & Moxidectin ${ }^{3}$ & 24 & Fort Myers, FL \\
\hline Sp 1 & 905 & 19.6 & $>40$ & $>1000$ & $>1000$ & Moxidectin ${ }^{3}$ & 23 & $\mathrm{FL}$ \\
\hline Sp 4 & 1125 & 6.1 & 16.9 & NT & NT & Pyrantel $^{6}$ & 23 & FL \\
\hline Sp 5 & 2870 & 6.9 & 31.5 & $>1000$ & $>1000$ & Unknown & 23 & FL \\
\hline Sp 6 & 560 & 7.3 & $>40$ & $>1000$ & $>1000$ & Moxidectin $^{3}$ & 23 & FL \\
\hline$\Delta \sim 1$ & $20 n$ & 95 & 101 & $>1 \cap \cap n$ & $>1 \cap n \cap$ & I Tnlennixin & ITnlennixn & FI \\
\hline
\end{tabular}


${ }^{7}$ For some samples the city of origin was not available. City/state refers only to the origin of the sample tested, but does not necessarily reflect the origin of the dogs prior to arriving at the respective kennels or veterinary practices.

* Pooled samples comprising ten dogs

NA: Assay data not available

NT: Assay data not available due to insufficient amount of eggs 